\title{
A Text Message a Day Keeps the Pulmonologist Away
}

\author{
Tae-Jung Yun \\ Samsung Electronics Co., Ltd. \\ Maetan 3-dong Yeongtong-gu \\ Suwon, Republic of Korea 442-600 \\ tj.yun@samsung.com
}

\author{
Rosa I. Arriaga \\ Georgia Institute of Technology \\ 85 Fifth Street NW \\ Atlanta, GA, USA 30308 \\ arriaga@cc.gatech.edu
}

\begin{abstract}
The goal of this study was to extend and replicate an SMS health intervention for pediatric asthma patients. This intervention was designed using the Health Belief Model (HBM). Thirty patients were randomly assigned to one of three conditions. In the Knowledge condition patients were queried about their asthma knowledge every other day. In the Knowledge and Symptoms condition patients received a daily text message. They were queried about their symptoms and knowledge of asthma on alternate days. The Control group received no texts. Our main finding is that daily text messages lead to improved health outcomes.

We explain our results in the context of interview data and the HBM. We conclude by suggesting that the HBM can be used to inform and evaluate system design for chronic care beyond asthma and by considering the role that replication studies can play in HCI research.
\end{abstract}

\section{Author Keywords}

Asthma; RCT; SMS; text message; RepliCHI

\section{ACM Classification Keywords}

H.5.m. Information interfaces and presentation (e.g., HCI): Miscellaneous.

\section{General Terms \\ Human Factors; Design; Measurement.}

\section{INTRODUCTION}

Chronic disease is the leading cause of death in the United States. Nearly fifty percent of the American population has at least one chronic disease and $21 \%$ have multiple chronic diseases [19]. The economic toll is severe, with some estimates indicating that up to $75 \%$ of health care costs in the United States are related to chronic care expenditures $[12,26]$.

HCI researchers have developed a number of mobile health technologies aimed at curbing the negative toll that chronic illnesses cause and improving health outcomes for patients. Both user studies [13,21,36] and theories of behavior change (e.g., [11]) have played a role in the development of

Permission to make digital or hard copies of all or part of this work for personal or classroom use is granted without fee provided that copies are not made or distributed for profit or commercial advantage and that copies bear this notice and the full citation on the first page. To copy otherwise, or republish, to post on servers or to redistribute to lists, requires prior specific permission and/or a fee.

CHI 2013, April 27-May 2, 2013, Paris, France.

Copyright (C) 2013 ACM 978-1-4503-1899-0/13/04...\$15.00. health interventions.

In this paper, we replicate and extend the result of a previous study [39]. This intervention was designed using the Health Belief Model, a cognitive theory of how individuals come to engage in preventative care management of their illness. In the current random control study, text messages were sent to test the hypothesis that increasing the child's awareness of his or her symptoms and improving knowledge of asthma would improve the child's health outcome. In addition, we tested the hypothesis that knowledge texts sent every other day could improve health outcomes. We collected physiological, psychological, and interview data.

The main results of the current study are that daily text messages improved patients' perceived severity of their disease and their awareness of their symptoms, and led to improved pulmonary function and quality of life. We end the paper by discussing the results in light of the replicated work, interview data, and the HBM.

\section{BACKGROUND}

Our research has focused on asthma. Asthma is a chronic disease that affects the lungs. It is a complex disorder characterized by variable and recurring symptoms. The World Health Organization (WHO) has estimated that over 200 million people suffer from asthma and that it is the most common childhood disease [38]. It affects over 5 million children in the United States [1].

Asthma has dire consequences for children beyond their physical health. It is responsible for the most school absences caused by a chronic condition [37]. Some researchers have argued that this leads children to have an academic handicap [6,22,25]. Beyond the psychosocial effects of having a chronically ill child, asthma has a negative economic toll on families. Up to $30 \%$ of parents report missing work because of their child's asthma.

\footnotetext{
Lessons learned from previous study with asthmatic patients

Information and communication technologies (ICT) have been developed and used to support asthma care selfmanagement. Europe leads the way in these kinds of interventions. In this section we present studies that had a mobile or web interface where the goal was to improve health outcomes through ICT intervention.
} 
Studies have been conducted using both observational and random control methodologies. The outcomes of interest include psychological (e.g., quality of life questionnaires) and physiological (lung function tests) measures. Three observational studies in the UK found increased selfefficacy with the use of Short Message Service (SMS, i.e, texting) technology. Neville et al. had thirty patients enrolled for a one-month period. The intervention consisted of simple medication reminders via their cell phones [24]. During focus groups users reported that the texts helped them comply with daily medication regimens. Ryan et al. sent patients texts concerning their peak flow values (maximum speed of expiration) [32]. Patients reported that the texts made them better able to manage and monitor their asthma. In another study, 9 participants indicated that they found mobile phone technology empowering; however, some wondered if this approach might engender dependence on reminders [28].

Researchers in Denmark have conducted extensive longerterm observational and experimental studies related to asthma. In one study, patients were asked to enter information concerning their asthma symptoms and medication into a web-based asthma diary [3]. In this study, physicians were able to access patients' information online. Results indicate the two-month deployment led to improved interaction between physicians and patients. This was tempered by the fact that technology use diminished over time. The researchers proposed that this was the case because Internet use was not part of the patient's everyday life. The researchers noted that the main problem with this technology design was that it was a passive tool rather than one that actively solicited information from the patients. A follow-up study addressed the latter issue by soliciting asthma diary information via mobile phones text. After a two-month period participants reported that they found the reminders about medication usage helpful and had a better sense of control [3]. This study, however, did not verify the patient's asthma diagnoses or include a physiological outcome measure.

The largest ICT study to date was a clinical trial with 300 asthma patients who were randomly assigned to one of three monitoring groups: Internet-based; a specialist; or a general practitioner [30]. Results indicated that over a sixmonth period patients in the Internet-based group (compared to the other two groups) did better on the quality of life index, lung function measure, and airway responsiveness measure. Another Internet-based random control trial showed increased quality of life and symptom relief but not lung function improvement [15]. One of the limitations of these studies is that they required intensive interaction from clinical personnel.

In a series of recent randomized control trials, positive outcomes have been reported with SMS interventions but physiological improvement has been mixed. In one study with 26 patients, researchers were able to increase adherence to medical regimen (but not improve health outcomes) when they sent text messages reminding them to take their medication [35]. Another study did report improved lung function after a 16-week randomized controlled trial with 16 young adults [27]. However, this study relied on intensive clinical support to the patient. Health care providers would adjust the individual patient's medication based on weekly reviews of the text messages they received from patients. The revised information would be sent back the patient via text message.

These past studies led us to a set of requirements to drive the technological implementation and methodology of our studies. First, we opted for a mobile (versus internet-based) platform because it is a natural technology to use with pediatric patients. In particular, SMS interventions have been successful in improving both psychological and physiological health outcomes. [20,34]. We decided to use a randomized control methodology because it is the only way to draw causal inferences about the effects of an intervention.

Additionally, our studies bridged several gaps in the literature we reviewed. We involved pediatric asthma patients (children 17 years and younger) previous studies were only conducted with adults. We simplified the interaction required from users to a single key press. The SMS intervention was simplified so that queries were part of a static database (e.g., not individualized) and so that no feedback was required from health care providers.

\section{THEORETICAL RATIONALE FOR THE INTERVENTION}

Our goal is to improve pediatric patient outcomes. Central to this goal is the hypothesis that the child will engage in positive asthma management practices. The HBM explains and predicts positive health-related behavior in terms of the psychological and experiential factors that lead to positive behavior change (see Figure 1).

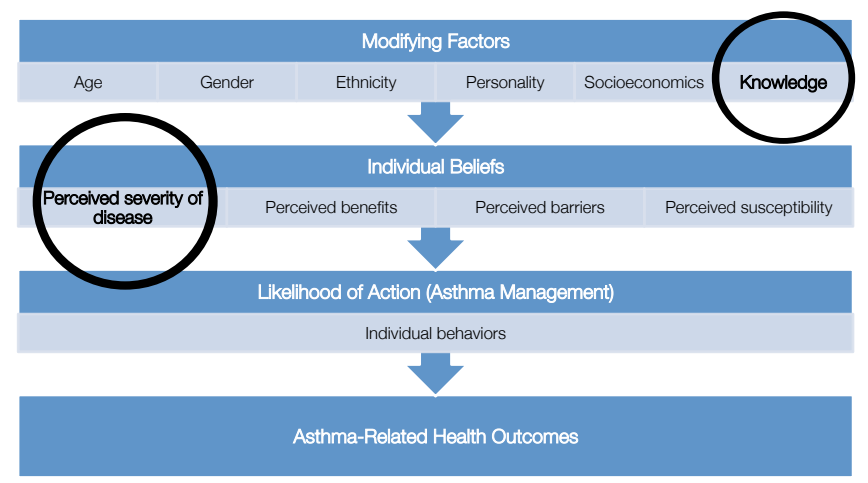

Figure 1: Health Belief Model adapted to asthma with variables of interests circled.

The model is compelling because its principles are relevant to both physiological and psychological conditions $[4,5,16,31]$. Specifically, the HBM argues that several factors influence whether an individual engages in beneficial health behavior. Taking into account previous 
literature and practices in the field, we focus on two variables: "Perceived Severity of the Disease," which is a personal belief; and "Knowledge," which is a variable that can help modify the individual's personal belief. The HBM highlights the role of the individual in any behavior change. Therefore, we have similarly prioritized the patient's role in asthma management and delivered messages directly to each patient (as opposed to the child's parent, which is the case with some standardized questionnaires [33]).

We conceptualized that one way to improve the Perceived Severity of the Disease would be to increase the patients' awareness of the deleterious effects that their symptoms were causing them by providing specific queries. We used a subset of questions from a preexisting questionnaire [33] and other questions that our clinical partners thought were important. We endeavored to increase the patients' perceived severity of their disease by having them reflect on the symptoms they were experiencing and the changes that these symptoms caused in their asthma management (use of rescue medicine or stronger medicine). Specifically, by asking the patient about asthma symptoms and management, we raise his awareness of potential discomfort or inconvenience that his condition is causing him.

In addition to making the patient aware of his symptoms, we planned to increase the patient's knowledge about asthma. This decision is supported by prior literature, which suggests that educating the pediatric patients themselves can result in improved health outcomes. Specifically, one recent study reported that, relative to usual care, pediatric asthma education was associated with reductions in the mean number of hospitalizations and emergency department visits [10].

The HBM underscores the importance of enhancing individual beliefs through effective communication channels that are relevant to the specific population. This reinforced our decision to use an SMS-based system, because it is the communication mode of choice for youths in this age range [20]. In considering how to increase the chances that the patients will acquire new knowledge about their condition, we capitalize on research from the area of learning. Microlearning refers to learning things in digestible small chunks [14]. The mobile phone lends itself well to using this knowledge acquisition technique because of the limited information that can be presented (on a small screen) and the way it allows us to intermittently access information [23].

\section{STUDY MOTIVATION AND DESIGN}

We have used the HBM to inform the design of our interventions. Specifically, we hypothesized that the two factors that were likely to lead to improved health outcomes were symptom awareness and knowledge acquisition (see Figure 1).

\section{Study 1 Review}

Patients were recruited from a private pediatric asthma clinic in the suburbs of an American city. The clinic serves over 15,000 patients and is one of the largest private practices in the country. Patients in this practice have been diagnosed as having asthma that is categorized as moderate to severe and thus requires care from specialists [28].

Thirty pediatric patients (10-16 years of age) were randomly assigned to one of three conditions: control (C) group, e.g., received no text messages; symptom (S) awareness condition (one text on alternate days); or symptom awareness and knowledge (S\&K) condition (one text a day alternating between $\mathrm{S} \& \mathrm{~K}$ ). In line with our goal to develop a simple intervention, we developed fifteen $S$ and $\mathrm{K}$ queries using resources provided by our clinical collaborators. The queries were presented in a static fourweek template for the duration of the study (see [39] for detailed rationale).

Once patients received the texts they were required to reply with a single key response (see Figure 2). In the case of the knowledge queries, the response would automatically trigger another text that provided feedback about the accuracy of their response and then present them with a factoid about asthma.

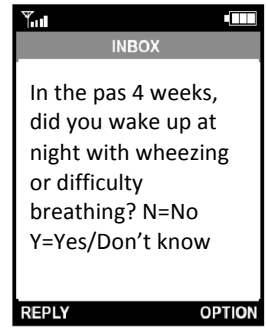

(A)

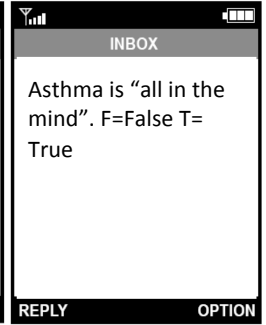

(B1)

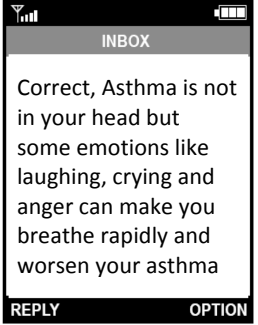

(B2)
Figure 2: Example of $S(\mathrm{~A})$ and $K(\mathrm{~B} 1, \mathrm{~B} 2)$ texts.

Analyses indicated no pre-existing differences between individuals assigned to each of the three conditions, and thus the random assignment protocol was successful. The main results from this study were that: 1) patients in $\mathrm{S}$ condition did not show improved health outcomes; and 2) patients in the S\&K condition showed clinically ${ }^{l}$ and statistically improved lung function (Forced Expiratory Flow (FEF) 25-75\%) after the study period compared to the $\mathrm{C}$ group $(t(9)=2.04, p=.036$, Cohen's $d=1.36$, effectsize $r=0.56$ ). Patients in the texting conditions compared to the control condition showed improved quality of life as measured by a standardized psychological scale for pediatric asthma patients [17].

\footnotetext{
${ }^{1}$ The change in values was regarded by the clinician as leading to ameliorated asthma symptoms
} 
Study 2 Replication and Extension of Previous Findings The results from Study 1 bolstered the notion that health outcomes could be improved with daily text messages. However, there were a number of issues that limited the inferences that could be drawn from it. First, while the initial study had a large effect size, the small sample size raised the question of whether or not the findings could be replicated with a new set of patients. Second, while Study 1 showed that $\mathrm{S}$ queries alone did not lead to lung function improvements, we did not know if the synergistic effects of S\&K were responsible for the outcomes or whether $K$ queries were driving the effect. Thus, we decided to replicate the original condition $(\mathrm{S} \& \mathrm{~K})$ and to systematically evaluate the effect of $\mathrm{K}$ alone.

\section{Participants}

Thirty pediatric asthma patients from the same clinical practice as those of Study 1 were enrolled. We used the same inclusion criteria in both studies. Specifically, pediatric asthma patients had to have visited the clinic on a previous occasion, be at least 10 years of age, have their own mobile phone, and be able to read at a fifth grade level. Additionally, patients from Study 1 were excluded from participating in Study 2. One of the patient's parents and the patient's pulmonologist were also enrolled.

\section{Method}

The protocol and list of texts sent to patients were the same between this study and Study 1 [39], including the list of S and $\mathrm{K}$ queries that were sent to all patients. Patients were randomly assigned to one of three groups. In the $\mathrm{S} \& \mathrm{~K}$ condition patients received a text message every day that alternated in content between $\mathrm{S}$ and $\mathrm{K}$ queries. Patients in the $\mathrm{K}$ condition received queries every other day. The study deployment period was the time between two scheduled clinic visits. This was the case because the "Guidelines for the Diagnoses and Management of Asthma" recommends visiting the doctor three to four times a year [29].

At the first medical visit, we provided parents with consent forms and patients with assent forms. A variety of measures were utilized (see Table 1). Due to space constraints, we focus on a subset of psychological and physiological measures. Parents were given the Pediatric Asthma Caregiver Quality of Life Questionnaire (PACQLQ) [18] to measure the problems they perceive as most troublesome. Patients were given the Pediatric Asthma Quality of Life Questionnaire (PAQLQ) [17], which assesses the functional problems (physical, emotional and social) they encounter because of their asthma. They were asked to report the data from their pulmonary function test, the FEF25-75\%. This was conducted by clinical staff and provides a physiological indicator of the patient's asthma status [17]. Patients were told that their physicians would be able to view the responses they sent via SMS. Pediatric patients who enrolled received $\$ 25$ after completing a series of pre-study materials and another $\$ 25$ after completing the post-study protocol (see Table 1).

\begin{tabular}{|l|l|l|l|}
\hline & $\begin{array}{l}\text { 1st Medical } \\
\text { Visit }\end{array}$ & $\begin{array}{l}\text { Between } \\
\text { Visits }\end{array}$ & 2 $^{\text {nd }}$ Medical visit \\
\hline Patient & -Pre-surveys & SMS log & -Post-survey \\
& -Technology & & -Asthma knowledge \\
& test \\
& -Asthma & & -ATAQ \\
& knowledge test & & -PAQLQ function \\
& -ATAQ & & -Pulmonary fata, FEF25-75\% \\
& -PAQLQ & & -Interview \\
& -Pulmonary & & \\
& function data, & & \\
& FEF25-75\% & & \\
\hline Parent & Pre-survey & & Post-survey \\
& -PACQLQ & & PACQLQ \\
& & & Interview \\
\hline
\end{tabular}

Table 1: Measures collected during the study.

During the second medical visit we collected the same measures. Additionally, patients and parents in the study groups were interviewed. Although parents and children were interviewed separately, parents stayed in the same room and were able to listen to their child's responses. This may have affected what the child said. The semi-structured interview focused on the participants' general experiences with the interventions, how they managed asthma during the study, and how they talked to their healthcare provider during the follow-up visits. They were asked questions about whether they were satisfied with the text message system. Interviews were recorded and transcribed for further analysis.

One author and another independent HCI researcher analyzed the interview transcripts using thematic analysis [7]. The reason we used thematic analysis was its flexibility. We followed the four phases of analysis. First, we generated inductive initial codes from the transcripts. These codes identify features of the interview data. Second, after finding and collating the codes, we searched for themes. We refocused the codes at the broader level and collected relevant codes within the interesting themes. At this point, we ended up with a collection of candidate themes. Next, we reviewed and refined the themes identified in the previous phase. We identified evident themes while merging or separating other themes. Finally, we further refined our themes and then collected interview quotes for each theme.

\section{RESULTS}

\section{Baseline at the first medical visit}

No meaningful differences were found in either clinical or psychosocial characteristics between the three experimental conditions. This indicates that the random assignment protocol was successful.

\section{Quantitative Outcome at the 2nd medical visit}

Complete $\left(1^{\text {st }}\right.$ and $2^{\text {nd }}$ visit) data was collected for 21 pediatric asthma patients (See Table 2). The time between 
two visits ranged from 67 to 148 days (median=118, mean=115 days). This sample size and duration of our study was similar to other SMS studies [27,28,35].

The SMS service sent a total of 2095 queries to 30 pediatric patients who were in the two experimental groups. The average response rate of 21 participants, who completed the study was $85.6 \%$ (Median 91.3\%), ranging from 36\% to $100 \%$.

\begin{tabular}{|l|l|l|l|}
\hline \multicolumn{4}{|c|}{ EXPERIMENTAL CONDITIONS } \\
\hline & Control & Knowledge & $\begin{array}{l}\text { Symptoms/ } \\
\text { Knowledge }\end{array}$ \\
\hline Girls & 4 & 5 & 3 \\
\hline Boys & 2 & 3 & 4 \\
\hline Age & $12.3 \pm 0.7$ & $14.0 \pm 0.7$ & $12.0 \pm 0.7$ \\
\hline
\end{tabular}

Table 2: Participant Information at $2^{\text {nd }}$ Visit.

Data (Table 2) indicated that patients in the S\&K condition compared to the $\mathrm{C}$ condition had both improved lung function $(t(11)=1.41, p<0.09$, Cohen's $d=0.85$,effectsize $r=0.3912$ ) and quality of life as measured by the meaningful improvement of PAQLQ, based on the Minimal Important Difference (MID); 0.56 \pm 0.34 vs. .50). Patients in the Knowledge group did not have better health outcomes compared to the control group.

There was support for the notion that the HBM variables, knowledge of asthma and awareness of the severity of their symptoms, affected the experimental groups beyond the health outcomes we measured. First, we conducted a asthma knowledge test, which we created, at the first and second medical visit and found that compared to the $\mathrm{C}$ group both the K group $(t(7)=4.25 p=0.0019$, Cohen's $d$ $=3.213$, effect-size $r=0.849)$ and the S\&K group had statistically significant improvement in knowledge $(t(6)=$ $2.2 p=0.035$, Cohen's $d=1.799$, effect-size $r=0.669$ ).

Second, to evaluate our conceptualization that raising the patient's awareness of their symptoms (through text messages) would increase their perceived severity of their illness, we conducted another evaluation. We compared children's perception of their asthma severity against their lung function test at both the first and second visits. We did this by asking children to categorize the severity of their asthma using the following scale: 1) Intermittent - have symptoms 2 or less days per week; 2) mild - have symptoms more than 2 days per week; 3) moderate - have symptoms daily; and 4) severe - have symptoms throughout the day. (These four categories come from national guidelines for evaluating asthma severity [29]). Their response was then correlated using Spearman's $\rho$ to their lung function measure. These measures were inconclusive at the initial visit for all of the experimental groups. However, during the second visit, only the S\&K group had a statistically significant correlation between their perceived severity ranking and their pulmonology function FEF25-
$75 \%(\rho=-0.8214 ; p=0.0234)$. Thus, patients in the S\&K group showed a more accurate perception of the severity of their asthma then patients in the $\mathrm{K}$ or $\mathrm{C}$ groups.

\section{Interview Analysis}

Both patients and their parents were interviewed after the medical visit. The interview data was analyzed with two goals in mind: to understand patients' usage of the SMS system; and to understand and patients' and parents' perceptions about the impact that the SMS system had on the patient's asthma management.

\section{Emergent Themes from the parent-child interviews}

Figure 3 shows the four themes that we extracted from the parent-child interview data concerning the effect that the SMS system had on the patient's asthma management. We first discuss how asthma management changed because of the SMS system. Second, we describe how the intervention affected knowledge. Third, we explain how the intervention influenced the perceived asthma condition. Next, we show how the intervention facilitated communication. Finally, we discuss the experience of participants with the SMS service.

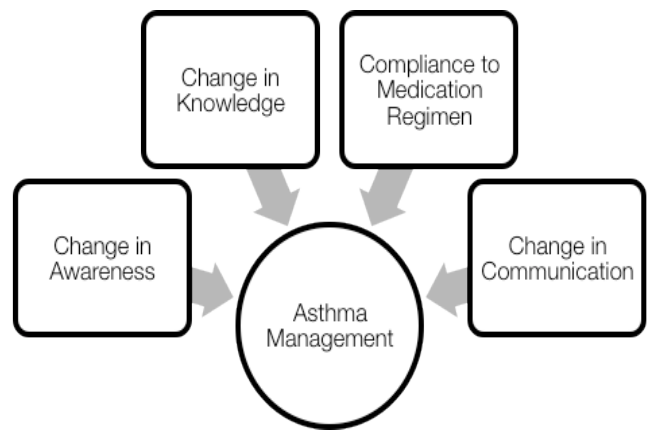

Figure 3: Emergent Themes from the Interview Data.

Theme 1: Change in Asthma Awareness

Interview data from participants in the S\&K group (and their parents) indicated that the SMS messages did in fact make them reflect on the symptoms they were having because of their asthma. They said that when they had texts on $\mathrm{S}$ query days they tried to recall if they had had a given symptom or had to alter their medication because of asthma exacerbation:

B4 SK-C " Umm, I think I thought about what the problems I was having more if any problems. I like what can I do to get this better... I think after the questions may be I feel lucky I don't have any problems since the study.'

G10 SK-C "In the past few days, has this, this, or that happened, you know. I would think about it, and it really let me think about it. Wow! that really did happen. You know I didn't even think about that or realize (without the questions).”

2 Interview quote starts with $\mathrm{B}$ (male participant) or $\mathrm{G}$ (female participant), then $\mathrm{K}$ (Knowledge group) or SK (Symptom \& Knowledge group) - C(child) or P (parent). 
B11 SK-P "It made him more aware. He did a lot better it increased awareness.”

It is worth noting that none of the patients or parents in the knowledge condition mentioned that their symptom awareness had changed over the duration of the study.

\section{Theme 2: Change in Asthma Knowledge}

Both children and parents agreed that the text messages led to improved knowledge about asthma. They noted that this made them feel better.

G4 K-C “(I) feel better like I knew what I was doing. Cause I think that I got fewer wrong which maybe feel better."

G15 K-C "Some of the question I didn't know because I've not been really told about."

G15 K-P 'It's been very informative. There have been a couple of times we had previous assumptions about things changed. She's answered the question wrong. It sent the correct information. And there has been a couple of questions that we had one belief, and the text message corrected what we had previously thought."

The interview data suggest that the format of the knowledge queries was appropriate. They viewed the repetition as serving to reinforce what they knew or had learned. Some questions were relatively easy for older children; however, the older children were not universally dissatisfied with these questions. Some patients said that these questions helped them confirm what they knew; while others stated that they wanted to receive more difficult questions.

\section{Theme 3: Compliance to Medication Regimen}

Children and parents mentioned that there was an increase in the regularity with which the child took the medicine, and that this was due to the text messages they received. It seems that the texts worked as reminders to take medication. In the language of the HBM, receiving and answering the text messages was a "cue to action." While the text messages in the SMS service did not have any content that explicitly reminded them to take their medication, the analysis suggests that patients used them this way. This was common to both the $\mathrm{K}$ group and the S\&K group. For example, two pediatric asthma patients set the text receiving time when they wanted to take their medication. One of them said:

B8 K-C "I woke up .. whenever I saw it,. Oh! I went to take my medicine."

Theme 4: Change in Communication

Receiving and answering the text messages seeded conversations among the various stakeholders. After receiving the text messages, children tried to initiate conversations. The interviews indicated that text messages increased the frequency and length of conversations about asthma between children and caregivers.
B11 SK-P "It made him discuss with his dad a lot because Dad doesn't know a lot about asthma. So kind of makes dad more aware too.",

The caregiver sometimes noted that there was less communication about asthma. This was perceived as a positive outcome because it showed that the child was more independent and less reliant on parent involvement with their asthma management:

G8 SK-P "I guess she and I didn't communicate a lot cause she's gotten better. I didn't hear any complaint."

The SMS service facilitated communication with the healthcare providers. During the follow up visits, the children reported having a better understanding about what the doctor was saying. It made them aware of what they did not know and more inquisitive about their asthma:

G15 K-C "(It) makes me have more questions [for] the doctor"

Thus, receiving the knowledge questions when children did not know the answers triggered conversations with their caregivers and changed the quality of interaction with their doctors.

Other feedback about the SMS system

Pediatric asthma patients reported that they had an easy time interacting with the SMS system. This was evident in the high response rate that was maintained through the duration of the study. We expect that the system was not burdensome because texting is an essential part of these children's lives. The children indicated that they appreciated the limited number of messages and the simple response format:

G8 SK-C "It was easy. What I needed to do was just type the letters."

G10 SK-C "It was very simple, and it didn't take much at all to answer it."

\section{DISCUSSION}

This study was a replication and extension of a previous intervention with pediatric patients that had moderate to severe asthma [40]. The intervention lasted about four months, the typical time between two regularly scheduled visits to the clinic. In this study we found that daily text messages lead to improved psychological and physiological outcomes. In this section, we will first present the contributions of this work and then consider the implications for future work in the area.

This paper presents three main contributions to the $\mathrm{CHI}$ community for those who work in the area of chronic health. First, it encourages the use of ubiquitous technology for the primary stakeholder. Second, it promotes designing technology to both replicate and extend results. Third, it introduces researchers to a social theory of behavior change that can be used to design both interventions and technology. 


\section{Texting is ubiquitous}

We utilized SMS technology because it has been found to be a successful mode of intervention for adult asthmatic patients, and because research suggested that it is the communication mode of choice for youths in America. Our findings echoed these results. In both Study 1 and the current study, we had a median response rate of over $85 \%$ over the four-month duration of the study. Pediatric patients reported that responding to the text messages was effortless and second nature to the way they communicate. It is worth noting that the response rate was high even when they found that the content of the messages was boring because of the repetitive content.

Previous SMS and Internet studies [30] that showed improved health outcomes required extensive engagement from the patients and at times from the health providers. In one study, patients had to text a diary entry about their asthma [3]. In another study, patients entered data about their respiratory health multiple times a day. A health provider would then review this data on weekly basis and then provide personal feedback to each patient. In our current study, all patients received the same messages and the only interaction that was required from them was a single key response. This study and our previous study [40] provide conclusive evidence that even simple interventions can lead to improved short-term psychological and physiological outcomes.

We underscore the point that our results show short-term positive outcomes. It may be the case that tailored texts or more interactive texts are necessary to keep patients engaged for longer-term deployments.

\section{Do more texts lead to better health outcomes?}

One of the goals of this study was to extend what we had learned from our previous SMS study [40]. In our previous study we found that the $S \& K$ condition lead to improved psychological and physiological health outcomes, but that this was not the case for patients in the $\mathrm{S}$ condition (even though we utilized the same set of symptoms queries in both conditions). Thus, for us the overarching question was, are knowledge queries driving the positive outcomes we witnessed in the $S \& K$ condition? In order to test this hypothesis we included a $\mathrm{K}$ condition in this study.

In this study we found that patients that received knowledge queries every other day did not have improved health outcomes. This is despite the fact that data from Study 1 and the present study found that knowledge queries result in increase in asthma knowledge from pre- to postintervention. This has been found in other chronic care studies as well [21].

One explanation for why daily text messages led to an improvement in health outcomes compared to those delivered on alternate days is that more messages are better irrespective of the content. While we have no data to counter this, we do have evidence that patients were attending to the messages themselves. Specifically, in Study 1 patients in the $\mathrm{S} \& \mathrm{~K}$ condition but not the $\mathrm{S}$ or control condition improved their knowledge about asthma. In the current study we found that patients in both experimental groups (S\&K and $\mathrm{K}$ ) but not the control group improved their knowledge pre- and post-study. This provides evidence that patients were attending to the messages that were being sent. One way to test the "more is better" hypothesis using our conceptualization of the HBM is to include a SS condition where patients get symptom messages every day. A related hypothesis is "more interaction irrespective of content is better.". In other words, the results have nothing to do with the patients receiving messages about their symptoms or asthma knowledge but everything to do with feeling connected to the healthcare provider. Here the arduous task would be to find the appropriate "dummy content" to include in the texts.

\section{HBM as a lens for design and assessment}

In this paper we propose that the Health Belief Model (HBM) can be used to develop interventions that lead to improved health outcomes. We have done this by designing interventions that utilize HBM constructs (e.g., symptoms and knowledge, see Figure 4). For example, in this and other studies we have examined whether the Perceived Severity of Disease can be heightened by making the patient aware of the negative effects of their symptoms via SMS queries.

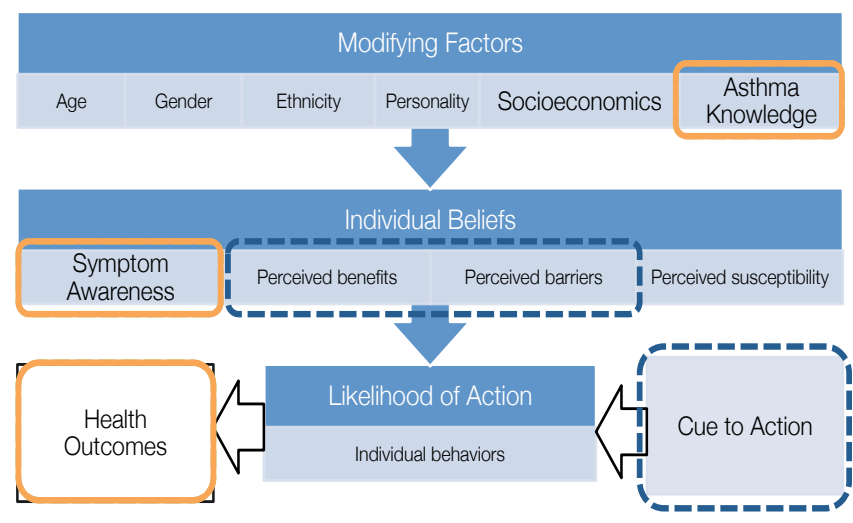

Figure 4: HBM Model variables that were evaluated in this study are presented with solid lines and those that emerged are presented with a dashed line

In the current study, we received both qualitative and quantitative support for this notion. First, interview data indicated that both patients and their parents thought that SMS messages did affect their awareness of their asthma. We found that patients in the S\&K group showed a significant correlation between the reported severity of the patient's asthma (how often the patients were having symptoms) and their pulmonary function data during the second medical visit. The fact that this was not found to be the case for the other two experimental conditions suggests that the symptom queries were effective in improving 
patients' individual beliefs about the Perceived Severity of the Disease.

A second modifying variable from the HBM that was investigated in this study was knowledge. Here the hypothesis was that improved knowledge could lead to improved health outcomes. Although we found that patients in this intervention condition had improved asthma knowledge compared to the control group, they did not have improved health outcomes. The current study suggests that for patients from this population, the effect of knowledge on health outcomes maybe synergistic. In other words, patients needed to receive both knowledge and symptoms messages to garner health benefits. The open question is whether there is a population of asthma patients for whom knowledge queries alone can lead to improved lung function.

A third HBM construct, cue to action, emerged as another variable that may be relevant for chronic disease interventions. Cue to action refers to the effect that an intervention can have on proactive health behavior. The interview data suggests that the SMS messages served as both implicit and explicit reminders for patients to take their medicine. It was an implicit reminder in that the SMS messages themselves never explicitly encouraged patients to take their medicine. Yet some patients in this study (and our past study) reported that sometimes getting an SMS message reminded them that to take their medicine on days that they had forgotten to do so. For other patients, the SMS message was used as a "medicine alarm." They actually decided to set the time they received their text message to the time they wanted to take their medicine.

\section{Opportunities for future research}

While the current findings are encouraging, a number of methodological limitations must be considered. These include questions about the generalizability of the findings as well as the duration of the effect of the study. We take the position that in addressing the limitations of this study, we present opportunities for future research.

\section{Learning from limitations of the current study}

First, the generalization of our results may be limited by the fact that we conducted our studies in the same private practice. It can be argued that perhaps there is something about the profile of these patients that may render them more susceptible to the positive effects of the technology intervention, e.g., demographic homogeneity. In fact, all of the patients in this study had private health insurance, which may suggest that they come from a higher socioeconomic status. One way to address this limitation is to conduct this study with patients from the practice that have public health insurance or to conduct the study in a public health clinic. Another way to address this criticism of our study is to deploy this study in the wild. One can imagine allowing patients to enroll by giving them a number to text. The challenge there would be to collect the pre- and post- data that would allow us to ascertain that the intervention had improved health outcomes.

Beyond the generalizability of our study, there is the question of the duration of the effect. The current study shows that our study was effective over a four month period. Would this be the case over a longer period of time? One thing that the current interviews suggest is that a longer deployment would necessitate content development because some patients were board with the redundancy of the texts.

Another area for future research relates to the temporal resolution of children's memories when they answered the queries. Many of the $\mathrm{S}$ queries asked, "In the past 4 weeks did X happen?" Yet we never evaluated whether patients actually examined symptoms over the past month. Perhaps they only surveyed the last week. Knowing what the natural search space is for children's symptom recall could dictate the best way to modify the presentation of the texts to accurately capture the symptoms they encountered.

A third limitation of our findings is that the same researchers conducted the replication. We thus call for intra-lab collaboration. This call presents opportunities to consider how technology can be used to facilitate this endeavor. One solution is to put the system online and allow other researchers to use it. This can be done, but at that point we are presented with the conundrum of whether doing this kind of research now renders us medical researchers rather than HCI researchers. A question for the HCI community then is whether having impact in domain necessarily leads to this existential angst? When is a good time to move on from a given technology?

\section{Utilizing the $\mathrm{HBM}$ in other $\mathrm{HCl}$ interventions}

We have introduced the HBM model as a theoretical framework that may be useful to the HCI community that conducts health-related research. We focused on two constructs, but had others emerge in the context of our finding. The cue to action is a case in point. One suggestion is to experimentally test whether actively tethering the S\&K text queries to the time when the patient usually takes their medication leads to improved health outcomes. Similarly, the synergistic effects of the knowledge and symptom questions taken together can be related to other HBM constructs such as perceived susceptibility. Thus, our studies suggest that the HBM constructs can serve to inspire future studies in this design space. Research also indicates that testing a more complex version of the HBM, then we did, may offer greater predictive power [9].

The HBM is a dynamic model that has been utilized and modified by researchers in a variety of ways [9]. We used only two of the tenets of the traditional HBM [31] because we are interested in devising simple ICT interventions that are used directly by the pediatric patient. This however is far from an exhaustive or definitive use of the HBM. For example, other researchers may be more interested in designing for the child-parent dyad. These researchers may 
find the Children's Health Belief Model (CHBM) [8] a more powerful tool since one of its primary contributions is "its recognition of the influence of the child's primary caretaker."

We have argued that the HBM presents valuable opportunities for designing technology that helps pediatric patients manage their asthma. However, the true promise of the HBM, as we see it, is that it extends to the greater chronic health sphere. After all, it is a general theory of preventative health, not specifically about asthma. The HBM presents fertile ground for designing interventions and technology for other chronic diseases. The first study we would like to suggest is to replicate this study $(\mathrm{S}, \mathrm{K}$ and $\mathrm{S} \& \mathrm{~K})$ with another chronic pediatric illness. The hypothesis would be that increased awareness and knowledge could lead to improved health outcomes in, e.g., diabetes. The research would partner with a clinical practice, devise a set of symptom and knowledge questions and use relevant outcomes as dependent measures.

\section{CONCLUSION}

We have shown that simple SMS text messages delivered daily can lead to improved health outcomes for pediatric asthma patients. This is a compelling finding in light of the fact that it replicates previous results in our lab. We have shown that the HBM can be an important tool in designing and implementing interventions in the chronic disease space beyond asthma. Our results underscore the importance of technology that is both easy to use and ubiquitous for the primary stakeholder. Finally, our results support the idea that replication studies can lead to improvements in both system design and interventions, which can in turn lead to new insights.

\section{REFERENCES}

1. Akinbami, L.J., Analysis, O., Moorman, J.E., and Liu, X. Asthma prevalence, health care use, and mortality: United States, 2005-2009. National health statistics reports, 32 (2011), 1-14.

2. Anhøj, J. and Møldrup, C. Feasibility of Collecting Diary Data From Asthma Patients Through Mobile Phones and SMS (Short Message Service): Response Rate Analysis and Focus Group Evaluation From a Pilot Study. Journal of Medical Internet Research 6, 4 (2004), e42.

3. Anhøj, J. and Nielsen, L. Quantitative and Qualitative Usage Data of an Internet-Based Asthma Monitoring Tool. Journal of Medical Internet Research 6, 3 (2004), e23.

4. Becker, M.H. and Maiman, L. a. Sociobehavioral determinants of compliance with health and medical care recommendations. Medical care 13, 1 (1975), 10-24.

5. Becker, M.H., Radius, S.M., Rosenstock, I.M., Drachman, R.H., Schuberth, K.C., and Teets, K.C.
Compliance with a medical regimen for asthma: a test of the health belief model. Public health reports (Washington, D.C. : 1974) 93, 3 (1974), 268-77.

6. Bender, B.G. Are Asthmatic Children Educationally Handicapped?. School Psychology Quarterly 10, 4 (1995), 274-91.

7. Braun, V. and Clarke, V. Using thematic analysis in psychology. Qualitative Research in Psychology 3, 2 (2006), 77-101.

8. Bush, P.J. and Iannotti, R.J. A Children's Health Belief Model. Medical care 28, 1 (1990), 69-86.

9. Carpenter, C.J. A meta-analysis of the effectiveness of health belief model variables in predicting behavior. Health communication 25, 8 (2010), 6619.

10. Coffman, J.M., Cabana, M.D., Halpin, H.A., and Yelin, E.H. Effects of Asthma Education on Children's Use of Acute Care Services: A Metaanalysis. Pediatrics 121, 3 (2008), 575-586.

11. Consolvo, S., McDonald, D.D.W., and Landay, J.A. Theory-driven design strategies for technologies that support behavior change in everyday life. Proceedings of the 27th international conference on Human factors in computing systems - CHI '09, ACM (2009), 405-414.

12. Druss, B.G., Marcus, S.C., Olfson, M., and Pincus, H.A. The most expensive medical conditions in America. Health affairs (Project Hope) 21, 4, 10511.

13. Grimes, A., Kantroo, V., and Grinter, R.E. Let's play! ACM Press, New York, New York, USA, 2010.

14. Hug, T. Didactics of microlearning : concepts, discourses and examples. Waxmann, Münster, 2007.

15. Jan, R.-L., Wang, J.-Y., Huang, M.-C., Tseng, S.M., Su, H.-J., and Liu, L.-F. An Internet-Based Interactive Telemonitoring System for Improving Childhood Asthma Outcomes in Taiwan. Telemedicine and e-Health 13, 3 (2007), 257-268.

16. Janz, N.K. and Becker, M.H. The Health Belief Model: A Decade Later. Health Education \& Behavior 11, 1 (1984), 1-47.

17. Juniper, E.F., Guyatt, G.H., Feeny, D.H., Ferrie, P.J., Griffith, L.E., and Townsend, M. Measuring quality of life in children with asthma. Quality of Life Research 5, 1 (1996), 35-46.

18. Juniper, E.F., Guyatt, G.H., Feeny, D.H., Ferrie, P.J., Griffith, L.E., and Townsend, M. Measuring 
quality of life in the parents of children with asthma. Quality of Life Research 5, 1 (1996), 2734.

19. Kung, H.-C., Hoyert, D.L., Xu, J., and Murphy, S.L. Deaths: final data for 2005. National Vital Statistics Reports: From the Centers for Disease Control and Prevention, National Center for Health Statistics, National Vital Statistics System 56, 10 (2008), 1-120.

20. Lenhart, A., Ling, R., Campbell, S., and Purcell, K. Teens and mobile phones. Pew Internet and American Life Project. Pew Research Center. http://www. pewInternet. org/Reports/2010/Teensand-Mobile-Phones. aspx. Accessed August 8, 2010.

21. Mamykina, L., Mynatt, E., Davidson, P., and Greenblatt, D. MAHI: investigation of social scaffolding for reflective thinking in diabetes management. Proceeding of the twenty-sixth annual SIGCHI conference on Human factors in computing systems, ACM (2008), 477-486.

22. Moonie, S.A., Sterling, D.A., Figgs, L., and Castro, M. Asthma status and severity affects missed school days. The Journal of school health 76, 1 (2006), 18-24.

23. Moura, A. and Carvalho, A.A. Mobile Learning: Using SMS in Educational Contexts. In N. Reynolds \& M. Turcsányi-Szabó (Eds.). In Key Competencies in the Knowledge Society. 2010, 281-291.

24. Neville, R., Greene, A., McLeod, J., Tracy, A., and Surie, J. Mobile phone text messaging can help young people manage asthma. BMJ: British Medical Journal 325, 7364 (2002), 600-600.

25. Newacheck, P.W. and Halfon, N. Prevalence, impact, and trends in childhood disability due to asthma. Archives of pediatrics \& adolescent medicine 154, 3 (2000), 287-93.

26. Olin, G.L. and Rhoades, J.A. The five most costly medical conditions, 1997 and 2002: estimates for the U.S. civilian noninstitutionalized population. 2002.

27. Ostojic, V., Cvoriscec, B., Ostojic, S.B., Reznikoff, D., Stipic-Markovic, A., and Tudjman, Z. Improving asthma control through telemedicine: a study of short-message service. Telemedicine Journal and E-Health: The Official Journal of the American Telemedicine Association 11, 1 (2005), $28-35$.

28. Pinnock, H., Slack, R., Pagliari, C., Price, D., and Sheikh, A. Professional and patient attitudes to using mobile phone technology to monitor asthma: questionnaire survey. Primary care respiratory journal: journal of the General Practice Airways Group 15, 4 (2006), 237-45.

29. Program., N.A.E. and P. Expert Panel Report 3 (EPR-3): Guidelines for the Diagnosis and Management of Asthma-Summary Report 2007. The Journal of Allergy and Clinical Immunology 120(5 Supp, (2007), S94-138.

30. Rasmussen, L.M., Phanareth, K., Nolte, H., and Backer, V. Internet-based monitoring of asthma: A long-term, randomized clinical study of 300 asthmatic subjects. Journal of Allergy and Clinical Immunology 115, 6 (2005), 1137-1142.

31. Rosenstock, I.M., Strecher, V.J., and Becker, M.H. Social Learning Theory and the Health Belief Model. Health Education \& Behavior 15, 2 (1988), $175-183$

32. Ryan, D., Cobern, W., Wheeler, J., Price, D., and Tarassenko, L. Mobile phone technology in the management of asthma. Journal of telemedicine and telecare 11 Suppl 1, (2005), 43-6.

33. Skinner, E.A., Diette, G.B., Algatt-Bergstrom, P.J., et al. The Asthma Therapy Assessment Questionnaire (ATAQ) for children and adolescents. Disease management: DM 7, 4 (2004), $305-13$.

34. Solomon, M.R. Information technology to support self-management in chronic care: A systematic review. Disease Management \& Health Outcomes 16, 6 (2008), 391-401.

35. Strandbygaard, U., Thomsen, S.F., and Backer, V. A daily SMS reminder increases adherence to asthma treatment: a three-month follow-up study. Respiratory medicine 104, 2 (2010), 166-71.

36. Toscos, T. Using data to promote healthy behavior in children. Proceedings of the 9th International Conference on Interaction Design and Children IDC '10, ACM Press (2010), 344.

37. Wang, L.Y., Zhong, Y., and Wheeler, L. Direct and indirect costs of asthma in school-age children. Preventing chronic disease 2, 1 (2005), A11.

38. World Health Organization. WHO | World Health Statistics 2011. World Health Organization, 2011.

39. Yun, T.-J., Jeong, H.Y., Hill, T.D., et al. Using SMS to provide continuous assessment and improve health outcomes for children with asthma. Proceedings of the 2nd ACM SIGHIT symposium on International health informatics - IHI ' 12 , ACM Press (2012), 621-630. 